\title{
Leakage power reduction using the body bias and pin reordering technique
}

\author{
Jae Woong Chun ${ }^{1 \text { a) }}$ and Chien-Yi Roger Chen ${ }^{2}$ \\ ${ }^{1}$ Department of Electrical and Electronic Engineering, Anyang University, \\ Anyang-si, Gyeonggi-do, 430-714, Korea \\ 2 Department of Electrical Engineering and Computer Science, Syracuse University, \\ Syracuse, NY 13244, USA
}

a)jwchun@anyang.ac.kr

\begin{abstract}
This paper presents a new method to reduce the standby leakage power consumption using the body bias and pin reordering technique for nanometer-scale CMOS circuits. The proposed method, unlike the conventional reverse body biasing (RBB) technique, considers gate leakage to minimize the negative effects of the existing RBB approach. This minimization of the negative effects can be achieved by intelligently applying proper body bias to the appropriate CMOS network based on its status (on-/off-state) with the aid of a pin reordering technique. Experimental results on ISCAS' 85 benchmark circuits show that the proposed method can achieve improvements in terms of leakage power savings that range from $16 \%$ to $38 \%$ when compared with the previous works.
\end{abstract}

Keywords: leakage current, reverse/forward body bias, pin reordering, leakage power saving, nanometer-scale CMOS circuits

Classification: Integrated circuits

\section{References}

[1] A. Agarwal, S. Mukhopadhyay, A. Raychowdhury, K. Roy and C. Kim: IEEE Micro 26 (2006) 68. DOI:10.1109/MM.2006.39

[2] K. Roy, S. Mukhopadhyay and H. Mahmoodi-Meimand: Proc. IEEE 91 (2003) 305. DOI:10.1109/JPROC.2002.808156

[3] K. von Arnim, E. Borinski, P. Seegebrecht, H. Fiedler, R. Brederlow, R. Thewes, J. Berthold and C. Pacha: IEEE J. Solid-State Circuits 40 (2005) 1549. DOI: $10.1109 /$ JSSC.2005.847517

[4] J. Tschanz, J. Kao, S. Narendra, R. Nair, D. Antoniadis, A. Chandrakasan and V. De: IEEE J. Solid-State Circuits 37 (2002) 1396. DOI:10.1109/JSSC.2002. 803949

[5] A. Shrivastava, A. Saxena and S. Akashe: Frontiers of Optoelectronics 6 (2013) 338. DOI:10.1007/s12200-013-0348-4

[6] D. Lee, D. Blaauw and D. Sylvester: IEEE Trans. Very Large Scale Integr. (VLSI) Syst. 12 (2004) 155. DOI:10.1109/TVLSI.2003.821553

[7] D. Lee, W. Kwong, D. Blaauw and D. Sylvester: DAC (2003) 175. DOI: 10.1109/DAC.2003.1218934 
[9] A. Hokazono, S. Balasubramanian, K. Ishimaru, H. Ishiuchi, T.-J. K. Liu and C. Hu: IEEE Electron Device Lett. 27 (2006) 387. DOI:10.1109/LED.2006. 873382

[10] R. Brayton and A. Mishchenko: Computer Aided Verification (Springer, Berlin, 2010) 24. DOI:10.1007/978-3-642-14295-6_5

\section{Introduction}

With technology downscaling, subthreshold leakage current exponentially increases as threshold voltage $\left(V_{t h}\right)$ reduces. Previously, subthreshold leakage was the dominant leakage component; as a result, conventional leakage reduction techniques primarily focus on subthreshold leakage alleviation, whereas the effect of gate leakage current was neglected. However, downscaling of gate oxide thickness produces a significant gate tunneling leakage current, which has become a major leakage component in CMOS circuits $[1,2]$. Thus, the circuit-level techniques that were used to suppress subthreshold leakage must be reevaluated for nanometer-scale technologies because subthreshold leakage is not the only serious leakage source in the nanometer-scale era. Body biasing techniques have been introduced $[3,4,5]$ to manipulate the delay and leakage of the circuit by modulating the $V_{t h}$. The RBB is an effective method of reducing the subthreshold leakage in the standby mode by increasing the $V_{\text {th }}$ of CMOS transistors, and forward body biasing (FBB) has been used in active mode to increase the circuit performance by decreasing the $V_{t h}$ of CMOS transistors. Pin reordering technique $[6,7]$ is proposed to reduce gate leakage. The focus of our paper is on the applications of body biasing and pin reordering as the method for standby leakage power reduction. In this research, we analyze the RBB technique from a structural point of view and address the problems associated with overall leakage savings due to the lack of awareness of gate leakage. Additionally, there are inherent limitations of using the pin reordering approach. To overcome these problems and limitations in association with previous approaches (pin reordering and RBB techniques), we propose the leakage-aware body biasing method, which takes into account not only the subthreshold leakage but also gate leakage, resulting in enhancement of the efficiency of body biasing for leakage power reduction.

\section{Leakage-aware body biasing technique}

The RBB technique reduces the leakage power during standby mode by increasing the voltage of the PMOS body (N-well) with respect to the supply voltage $\left(V_{D D}\right)$ and by lowering the voltage of the NMOS body (P-well) with respect to the ground (Gnd), resulting in an increase in the $V_{t h}$, and thus suppressing the subthreshold leakage. In active mode, $V_{t h}$ decreased by removing the reverse body bias voltage $\left(V_{R}\right)$, thereby restoring the normal performance. From a structural point of view, however, the existing RBB technique does not take into account the impact of on-state networks of a CMOS circuit because $V_{R}$ is applied, regardless of the state status (on- and off-state) of the CMOS network; $V_{R}$ reduces the subthreshold leakage in an off-state network of CMOS circuits, whereas $V_{R}$ in an on-state 
network increases the gate leakage by increasing the voltage between the gate and body node. To illustrate this phenomenon, we analyze the components of steady state CMOS transistors in on- and off-state networks.

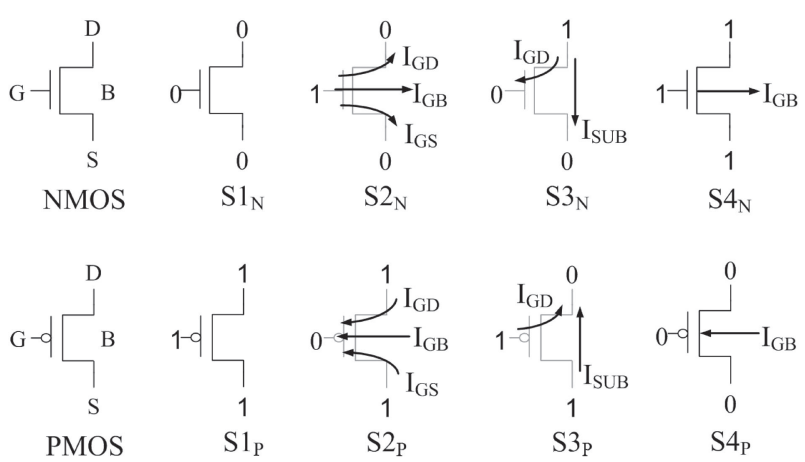

Fig. 1. Major leakage currents of typical steady states.

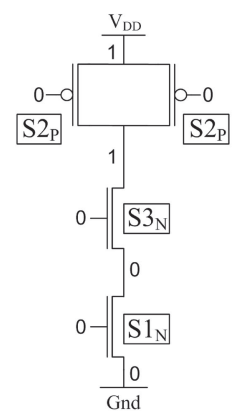

(a)

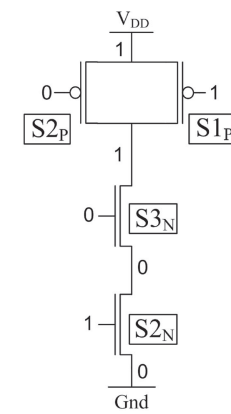

(b)

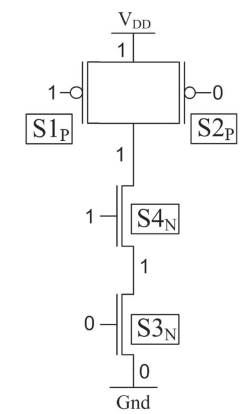

(c)

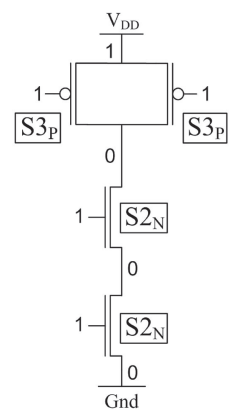

(d)

Fig. 2. Steady states of 2-input NAND gate.

Fig. 1 shows the major leakage currents (gate leakage current $\left(I_{G}=I_{G D}+\right.$ $\left.I_{G S}+I_{G B}\right)$ and subthreshold leakage current $\left(I_{S U B}\right)$ ) of typical steady states [8] in NMOS $\left(S 1_{N}-S 4_{N}\right)$ and PMOS $\left(S 1_{P}-S 4_{P}\right)$ transistors of basic logic gates (such as Inverter, NAND and NOR gates). The logic values " 1 " and " 0 " are denoted by the high and low level of each terminal node, respectively. A single transistor has four terminal nodes (gate $(G)$, drain $(D)$, source $(S)$ and body $(B)$ ), and the body of NMOS and PMOS are connected to Gnd (logic 0 ) and $V_{D D}$ (logic 1), respectively.

Among the steady states, the main steady state components of a CMOS network are $S 2_{P / N}$ and $S 3_{P / N}$ because these steady states are found in every CMOS logic gate, unlike other steady states. In the steady state of each CMOS logic gate, one network (either pull-up (PMOS) or pull-down (NMOS)) is always "on" and the other network is always "off", i.e., the on-state network always has at least one $S 2_{P / N}$, and the off-state network always has at least one $S 3_{P / N}$. In other words, every CMOS logic gate has either a pair of $S 2_{N}$ and $S 3_{P}$ or a pair of $S 2_{P}$ and $S 3_{N}$, depending upon its input vector of logic gate, as shown in Fig. 2. In addition, as shown in Fig. 1, $S 2_{P / N}$ and $S 3_{P / N}$ are the highest leaky states of on-transistors $\left(S 2_{P / N}\right.$ and $\left.S 4_{P / N}\right)$ and off-transistors $\left(S 1_{P / N}\right.$ and $\left.S 3_{P / N}\right)$, respectively, because the 
amount of leakage is a strong function of the voltage between the nodes. Hence, we use the main steady states $\left(S 2_{P / N}\right.$ and $\left.S 3_{P / N}\right)$ as typical examples to analyze the effects of $V_{R}$ on the on- and off-state networks.

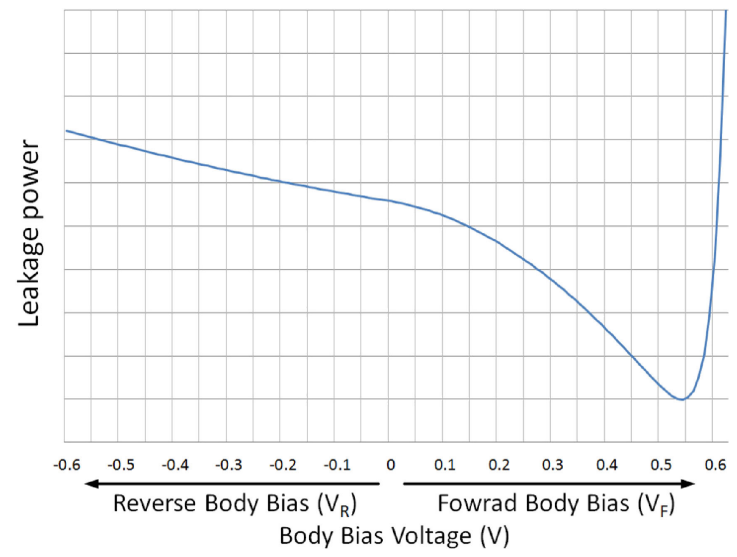

Fig. 3. Leakage power trends of $S 2_{N}\left(32 \mathrm{~nm}\right.$ with $\left.V_{D D}=0.9 \mathrm{~V}\right)$ as a function of the body bias voltage.

The on-state network of CMOS logic gates present either $S 2_{P / N}$ alone (e.g., Fig. 2(a) and (d)) or in combination with $S 1_{P / N}$ (e.g., Fig. 2(b) and (c)), and the other steady states are never present in an on-state network because the pull-up (pull-down) network is "on", and both source and drain nodes of every transistor in the on-state network present high (low) logic value, regardless of its gate input state. Note that the following analysis is performed for an NMOS transistor, but it is equally pertinent to a PMOS transistor. The impact of body bias voltage ( $V_{R}$ and forward body bias voltage $\left(V_{F}\right)$ ) on the leakage power of $S 2_{N}$ is shown in Fig. 3. It is shown that leakage power increased rather than decreased when $V_{R}$ is applied to $S 2_{N}$. This behavior is due to the fact that applying $V_{R}$ increases the voltage between the gate and body node $\left(V_{G B}\right)$, resulting in an increase of the gate leakage. Alternatively, when $V_{F}$ is applied to $S 2_{N}$, the leakage power of $S 2_{N}$ is decreased because the gate leakage decreased as $V_{G B}$ decreased by $V_{F}$. Note that $V_{F}$ yields the forward p-n junction leakage current between the body and drain/source node of $S 2_{N}$. Hence, the leakage power increased beyond $V_{F}=0.54 \mathrm{~V}$, as shown in Fig. 3. The maximum $\left|V_{F}\right|$ is conservatively assumed to be $0.6 \mathrm{~V}$ [9]. In this paper, the value of $\left|V_{F}\right|$ is set to the minimum point $(0.54 \mathrm{~V})$ of leakage power for a leakage savings in the on-state CMOS network.

The off-state network of CMOS logic gates present either the $S 3_{P / N}$ alone (e.g., Fig. 2(d)) or in combination with the other types of steady state, and thus, all types of steady states existed in the off-state network, as shown in Fig. 2(a), (b) and (c). The subthreshold leakage in the $S 3_{P / N}$ transistor is reduced as $V_{t h}$ increased by applying $V_{R}$ in the off-state network. An important point is that, unlike $S 3_{P / N}$, which only exists in an off-state network, $S 2_{P / N}$ exists in both an on-state network and an off-state network, as shown in Fig. 2(b). Therefore, the efficiency of reverse body biasing is reduced in the presence of $S 2_{P / N}$ in an off-state network. To take 
state network. Motivated by the above observations, we propose the novel leakageaware body biasing (LABB) method to maximize the standby leakage savings.

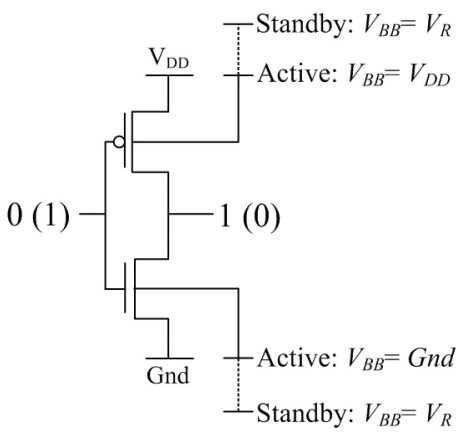

(a)

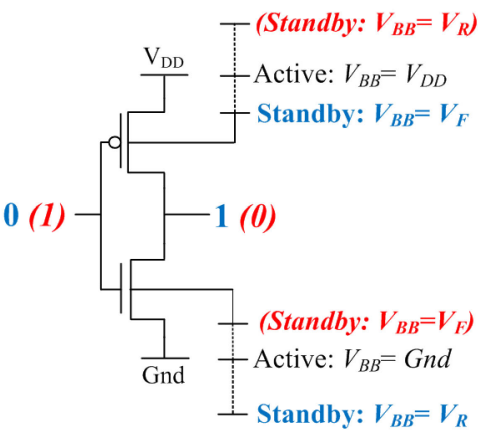

(b)

Fig. 4. (a) RBB technique (b) LABB technique

The RBB technique applied $V_{R}$ to each body of the CMOS network in standby mode, regardless of status of network, as shown in Fig. 4(a). With the understanding of the leakage behavior of the body bias voltage $\left(V_{B B}\right)$ in steady states, we first applied a proper $V_{B B}$ to the appropriate CMOS network based on its status in standby mode (i.e., $V_{F}$ and $V_{R}$ applied to the on-state network and the off-state network, respectively) as shown in Fig. 4(b) to remove the negative effects of $V_{R}$ on the $S 2_{P / N}$ in the on-state network, and we then use the pin reordering technique by placing every off-transistor in the pull-up/-down off-state network at the top/ bottom of the stack for each gate, resulting in exclusion of the $S 2_{P / N}$ from the off-state network; the pin reordering technique eliminates the $S 2_{P / N}$ in the off-state network by replacing the $S 2_{P / N}$ (e.g., Fig. 2(b)) with $S 4_{P / N}$ (e.g., Fig. 2(c)). For this reason, the proposed LABB method yields more leakage power reduction than RBB approach as shown in Table I which lists the gate leakage power $\left(P_{G}\right)$ and subthreshold leakage power $\left(P_{S U B}\right)$ savings $(\%)$ of 2-input NAND gate when different leakage reduction techniques are applied.

Table I. Leakage power savings (\%) of 2-input NAND gate

\begin{tabular}{|c|c|c|c|c|c|c|}
\hline \multirow{2}{*}{$\begin{array}{c}\text { Input } \\
\text { states }\end{array}$} & \multicolumn{2}{|c|}{ Pin reordering } & \multicolumn{2}{|c|}{ RBB } & \multicolumn{2}{c|}{ LABB } \\
\cline { 2 - 7 } & $P_{G}$ & $P_{S U B}$ & $P_{G}$ & $P_{S U B}$ & $P_{G}$ & $P_{S U B}$ \\
\hline 00 & 0 & 0 & -8.52 & 89.76 & 2.03 & 89.96 \\
\hline 01 & 35.01 & 37.26 & -7.68 & 94.15 & 36.94 & 94.26 \\
\hline 10 & 0 & 0 & -6.03 & 87.09 & 2.97 & 87.10 \\
\hline 11 & 0 & 0 & -1.11 & 88.84 & 0.56 & 88.85 \\
\hline
\end{tabular}

It should be noted that there is a limitation of using the pin reordering technique in CMOS logic gates because this technique is only applicable to certain cases of input state (i.e., the low input state (logic 0 ) is located above the high input state (logic 1) in off-state network) out of the $2^{n}$ possible input states in $n$-input logic 
gate. For instance, the pin reordering technique is only applicable to one input state ("01" in Fig. 2(b) and Table I) out of the four $\left(2^{2}\right)$ possible input states in 2-input NAND gate. On the other hand, the proposed method is applicable to all cases of input states. As seen in Table I, compared to the pin reordering technique, our method yields additional $P_{G}$ and $P_{S U B}$ savings by applying $V_{F}$ to on-state network and $V_{R}$ to off-state network, respectively.

In summary, the proposed LABB technique uniquely combines the advantages of two existing approaches, the pin reordering and body biasing techniques, to overcome the problems and limitations in association with the pin reordering and RBB techniques.

\section{Experimental results}

Table II. Experimental results for the ISCAS'85 circuits

\begin{tabular}{|c|c|c|c|c|c|c|}
\hline \multirow{2}{*}{ Circuits } & \multirow{2}{*}{$\begin{array}{c}\text { \# of } \\
\text { Gates }\end{array}$} & \multicolumn{3}{|c|}{$\begin{array}{c}\text { Average leakage } \\
\text { power savings (\%) }\end{array}$} & \multicolumn{2}{c|}{$\begin{array}{c}\text { Maximum leakage power } \\
\text { savings (\%) compared to }\end{array}$} \\
\cline { 3 - 7 } & & Pin reordering & RBB & LABB & Pin reordering & RBB \\
\hline C432 & 170 & 10.52 & 25.74 & 39.47 & 34.30 & 20.32 \\
\hline C499 & 366 & 7.04 & 21.41 & 37.63 & 32.07 & 19.14 \\
\hline C880 & 292 & 7.18 & 23.47 & 38.32 & 34.58 & 18.49 \\
\hline C1355 & 427 & 7.82 & 23.38 & 35.81 & 29.50 & 16.16 \\
\hline C1908 & 376 & 6.91 & 21.90 & 37.93 & 33.79 & 17.87 \\
\hline C3540 & 909 & 9.82 & 23.52 & 38.18 & 30.63 & 17.14 \\
\hline C6288 & 1999 & 9.14 & 25.70 & 41.61 & 37.97 & 17.63 \\
\hline \multicolumn{2}{|c|}{ Average } & 8.35 & 23.59 & 38.42 & 33.26 & 18.11 \\
\hline
\end{tabular}

The proposed leakage-aware body biasing method was implemented and evaluated for ISCAS' 85 benchmark circuits using a $32 \mathrm{~nm}$ predictive technology model. All circuits were synthesized using the Berkeley ABC tool [10] with Inverters, NAND $2 / 3 / 4$, NOR $2 / 3 / 4$, XOR2 and XNOR2 gates. The leakage power consumption for each circuit was measured for 1000 random input vectors using a HSPICE simulator. The experimental results are listed in Table II. The average leakage power savings of the existing techniques (pin reordering and RBB) and the proposed method are listed in columns 3-5. Columns 6 and 7 show the maximum leakage power savings of the proposed method when compared with the methods of pin reordering and $\mathrm{RBB}$, respectively. The results show that our approach achieves average leakage power savings of up to $41.61 \%$ (average: $38.42 \%$ ) for ISCAS' 85 benchmark circuits. The proposed method yields additional leakage power savings of up to $37.97 \%$ (average: $33.26 \%$ ) and $20.32 \%$ (average: $18.11 \%$ ) when compared with the methods of pin reordering and RBB, respectively.

\section{Conclusions}

In this paper, we demonstrated that the leakage behavior of body bias voltage in on-/off-state CMOS network is based upon the its input states. Then, we high- 
lighted the problems and limitations associated with existing approaches. To solve these problems, we presented the leakage-aware body biasing technique to minimize the leakage power consumption of nanometer-scale CMOS circuits in standby mode. The experimental results show that the proposed method improved the effectiveness of body biasing for leakage power reduction, confirming that the proposed method is an effective alternative method to the RBB technique. 